\title{
The Top Patient Safety Strategies That Can Be Encouraged for Adoption Now
}

Paul G. Shekelle, MD, PhD; Peter J. Pronovost, MD, PhD; Robert M. Wachter, MD; Kathryn M. McDonald, MM; Karen Schoelles, MD, SM; Sydney M. Dy, MD, MSc; Kaveh Shojania, MD; James T. Reston, PhD, MPH; Alyce S. Adams, PhD; Peter B. Angood, MD; David W. Bates, MD, MSc; Leonard Bickman, PhD; Pascale Carayon, PhD; Sir Liam Donaldson, MBChB, MSc, MD; Naihua Duan, PhD; Donna O. Farley, PhD, MPH; Trisha Greenhalgh, BM BCH; John L. Haughom, MD; Eileen Lake, PhD, RN; Richard Lilford, PhD; Kathleen N. Lohr, PhD, MA, MPhil; Gregg S. Meyer, MD, MSc; Marlene R. Miller, MD, MSc; Duncan V. Neuhauser, PhD, MBA, MHA; Gery Ryan, PhD; Sanjay Saint, MD, MPH; Stephen M. Shortell, PhD, MPH, MBA; David P. Stevens, MD; and Kieran Walshe, PhD

O ver the past 12 years, since the publication of the Institute of Medicine's report, "To Err is Human: Building a Safer Health System," improving patient safety has been the focus of considerable public and professional interest. Although such efforts required changes in policies; education; workforce; and health care financing, organization, and delivery, the most important gap has arguably been in research. Specifically, to improve patient safety we needed to identify hazards, determine how to measure them accurately, and identify solutions that work to reduce patient harm. A 2001 report commissioned by the Agency for Healthcare Research and Quality, "Making Health Care Safer: A Critical Analysis of Patient Safety Practices" (1), helped identify some early evidence-based safety practices, but it also highlighted an enormous gap between what was known and what needed to be known.

For the past 4 years, with support from the Agency for Healthcare Research and Quality, our group (a project team from the RAND Corporation; Stanford University; the University of California, San Francisco; Johns Hopkins University; and ECRI Institute) and an international panel of 21 stakeholders and evaluation methods experts conducted an evidence-based assessment of patient safety strategies (PSSs). Our efforts involved 3 phases. In the first phase, we developed a framework for reviewing existing studies and prospectively evaluating new PSS implementation studies (2). This framework identified several key points about the importance of theory, context, and implementation (Table 1) (2).

The second phase was a review of current patient safety strategies. We started with the 79 topics in Making Health Care Safer and added practices from the National Quality Forum's 2010 update, the Joint Commission, and the Leapfrog Group; those we identified in an initial scoping search; and those suggested by experts. From this list of 158 potential topics, we used several rounds of voting with our stakeholders to narrow the scope to 41 PSSs that the expert panel judged to be most important to the largest audience. Given limited time and resources, we prioritized topics as needing either a traditional systematic review or only a "brief review." The latter generally focused on a specific aspect of the PSS, such as emerging data or new insights about implementation.
We chose 18 topics for in-depth reviews. As a first step for the reviews, we searched for existing relevant systematic reviews. To assess the potential utility of such reviews, we followed procedures proposed by Whitlock and colleagues (3) and asked the following questions: Is the existing review sufficiently "on topic" to be of use? Is the review of sufficient quality to foster confidence in the results? If we determined that the existing systematic review was sufficiently on topic and of acceptable quality, we took 1 of 2 further steps. In some cases, we did an "update" search (that is, we searched databases for all new relevant evidence published since the search end date in the existing systematic review); in others, we conducted searches for "signals for updating." Such searches generally followed the criteria proposed by Shojania and colleagues (4), which involved a search of high-yield databases and journals for pivotal studies that could signal that a systematic review is out of date. A pivotal study is one that may call into question the results of a previous systematic review. We added any evidence identified in either the update search or signals search to the evidence base from the existing systematic review. Some PSSs had no existing systematic reviews and others had previous reviews that were not of sufficient relevance or quality to be used. In those situations, we conducted new searches using existing guidance (5).

Evidence about context, implementation, and adoption was a key focus of our reviews. We searched for evidence on these aspects of primary studies in 2 ways. First, we sought and extracted data about context, implementation, and unintended harms from articles that evaluated the effectiveness of PSSs. Second, we identified "implementation studies" from our literature searches. These studies focus on the implementation processes, particularly elements demonstrated or hypothesized to be of special importance for the success, or lack of success, of the intervention. To be eligible, implementation studies needed to

See also:

Web-Only

CME quiz (Professional Responsibility Credit) 
Table 1. Recommendations for Evaluating the Effectiveness of Patient Safety Strategies and High-Priority Contexts to Include in Reports of Patient Safety Research*

Recommendations for evaluating the effectiveness of patient safety
strategies
Explicitly describe the theory behind the chosen intervention components
or an explicit logic model for why this patient safety practice should
work
Describe the patient safety practice in sufficient detail so it can be
replicated, including the expected effect on staff roles
Measure high-priority contexts in the 4 domains described below
Detail the implementation process, the actual effects on staff roles, and
how the implementation or intervention changed over time
Assess the effect of the patient safety practice on outcomes and possible
unexpected effects, including data on costs, when available
For studies with multiple intervention sites, assess the influence of context
on the effectiveness of the intervention and implementation
High-priority contexts to include in reports of patient safety research
External factors, such as regulatory requirements, public reporting, or
pay-for-performance, and local sentinel events
Organization structural characteristics, such as size, complexity, and
financial status or strength
Teamwork, leadership, and patient safety culture
Management tools, such as training resources, internal organization
incentives, audit and feedback, and quality improvement consultants

${ }^{*}$ From reference 2 .

either report or be linked to reports of effectiveness outcomes.

The 23 brief reviews were explicitly designed not to be full systematic reviews or updates. The goals of each brief review varied by PSSs, according to needs identified by technical experts and stakeholders. The brief review could focus primarily on information about the effectiveness of an emerging PSS or implementation of an established PSS. Alternatively, the review could explore whether new evidence calls into question the effectiveness of an existing PSS or identifies unintended consequences of safety interventions. In general, a content expert on the topic, working with the project team, conducted the brief reviews. The methods involved focused literature searches for evidence relevant to the specific need. Typically, the author narratively summarized the evidence in a format tailored to the particular goal of the brief review.

We used standard instruments, such as the Cochrane Effective Practice and Organisation of Care criteria (6), the U.S. Preventive Services Task Force criteria (7), and the Cochrane Risk of Bias criteria (8), to assess the quality or risk of bias for individual studies of safety interventions. We developed criteria to evaluate strength of evidence across studies of effectiveness (9) that were informed by existing methods $(10,11)$ and incorporated criteria about the use of theory and description of implementation.

All of the reviews can be found in the Agency for Healthcare Research and Quality evidence report, "Making Health Care Safer II: An Updated Critical Analysis of the Evidence for Patient Safety Practices" (9). In this supplement issue, we present the reviews for 10 PSSs. In an upcoming issue of $B M J$

366 5 March 2013 Annals of Internal Medicine $\mid$ Volume 158 • Number 5 (Part 2)
Quality \& Safety, we will present several more. A summary of the evidence for all 41 PSSs is available in Table 1 of Chapter 44 in that report (9). It categorizes each PSS according to the following: the scope of the underlying problem that the PSS addresses (its frequency and severity); the strength of evidence about the effectiveness of the safety strategy; the evidence or potential for harmful consequences of the strategy; a rough estimate of the cost of implementing the strategy (low, medium, or high); and an assessment of the difficulty of implementing the strategy.

In the last phase of our effort, the expert panel explicitly considered the strength and quality of evidence about effectiveness and implementation for each PSS and concluded that 22 PSSs are ready to be encouraged for adoption by health care providers (Table 2). The first 10 are those that the expert panel believed should be "strongly encouraged" for adoption. The remaining 12 are ones they "encouraged" for adoption. Future implementation and evaluation will further our understanding of how best to implement these 22 practices to make them most effective and help health care organizations become learning health care systems. In the meantime, our expert panel believes that providers should not delay adopting these practices,

Table 2. Patient Safety Strategies Ready for Adoption Now

Strongly encouraged

Preoperative checklists and anesthesia checklists to prevent operative and postoperative events

Bundles that include checklists to prevent central line-associated bloodstream infections

Interventions to reduce urinary catheter use, including catheter reminders, stop orders, or nurse-initiated removal protocols

Bundles that include head-of-bed elevation, sedation vacations, oral care with chlorhexidine, and subglottic suctioning endotracheal tubes to prevent ventilator-associated pneumonia

Hand hygiene

The do-not-use list for hazardous abbreviations

Multicomponent interventions to reduce pressure ulcers

Barrier precautions to prevent health care-associated infections

Use of real-time ultrasonography for central line placement

Interventions to improve prophylaxis for venous thromboembolisms

\section{Encouraged}

Multicomponent interventions to reduce falls

Use of clinical pharmacists to reduce adverse drug events

Documentation of patient preferences for life-sustaining treatment

Obtaining informed consent to improve patients' understanding of the potential risks of procedures

Team training

Medication reconciliation

Practices to reduce radiation exposure from fluoroscopy and CT

The use of surgical outcome measurements and report cards, such as those from ACS NSQIP

Rapid-response systems

Use of complementary methods for detecting adverse events or medical errors to monitor for patient safety problems

Computerized provider order entry

Use of simulation exercises in patient safety efforts

ACS = American College of Surgeons; CT $=$ computed tomography; NSQIP $=$ National Surgical Quality Improvement Program. 
particularly the strongly encouraged ones. Enough is known now to permit health care systems to move ahead.

From the RAND Corporation, Santa Monica, Veterans Affairs Greater Los Angeles Healthcare System, Los Angeles, University of California, San Francisco, San Francisco, Stanford Center for Health Policy and Center for Primary Care and Outcomes Research, Stanford, Kaiser Permanente, Oakland, and University of California, Berkeley, Berkeley, California; Johns Hopkins Medicine Patient Safety and Quality, Johns Hopkins University, and John's Hopkins Children's Center, Baltimore, Maryland; ECRI Institute, Plymouth Meeting, RAND Corporation, Pittsburgh, and University of Pennsylvania, Philadelphia, Pennsylvania; Centre for Patient Safety, University of Toronto, Ottawa, Ontario, Canada; National Quality Forum, Washington, DC; Harvard University, Brigham and Women's Hospital, Boston, Massachusetts; Vanderbilt University's Peabody College, Nashville, Tennessee; University of Wisconsin-Madison, Madison, Wisconsin; Imperial College London and Queen Mary, University of London, London, University of Birmingham, Edgbaston, Birmingham, and Manchester Business School, University of Manchester, Manchester, United Kingdom; New York State Psychiatric Institute, New York, New York; PeaceHealth Medical Group, Eugene, Oregon; Research Triangle Institute International, Research Triangle Park, North Carolina; Dartmouth Institute for Health Policy and Clinical Practice, Lebanon, North Hampshire; Case Western Reserve University, Cleveland, Ohio; and Veterans Affairs Ann Arbor Healthcare System and University of Michigan, Ann Arbor, Michigan.

Note: The Agency for Healthcare Research and Quality reviewed contract deliverables to ensure adherence to contract requirements and quality, and a copyright release was obtained from the Agency for Healthcare Research and Quality before submission of the manuscript.

Disclaimer: All statements expressed in this work are those of the authors and should not be construed as official opinions or positions of the organizations where any of the authors are employed, the Agency for Healthcare Research and Quality, the U.S. Department of Health and Human Services, or the U.S. Department of Veterans Affairs.

Acknowledgment: The authors thank Aneesa Motala, BA.

Financial Support: From the Agency for Healthcare Research and Quality, U.S. Department of Health and Human Services (contract HHSA290-2007-10062I). Dr. Lilford was supported by the National Institute of Health Research Collaborations for Leadership in Applied Health Research and Care for Birmingham and the Black Country.

Potential Conflicts of Interest: Dr. Shekelle: Consultancy: ECRI Institute; Employment: Veterans Affairs; Grants/grants pending: Agency for Healthcare Research and Quality (AHRQ), Veterans Affairs, Centers for Medicare \& Medicaid Services, National Institute of Nursing Research, Office of the National Coordinator; Royalties: UpToDate. Dr. Pronovost: Board membership: Cantel Medical Group; Consultancy: Association for Professionals in Infection Control and Epidemiology, Hospitals and Health Care Systems; Grants/grants pending (money to institution): AHRQ, National Institutes of Health; Payment for lectures: Leigh Bureau (speaking on quality and safety); Royalties: Penguin Group. Dr. Wachter: Grant, support for travel to meetings, payment for writing or reviewing the manuscript, grants/grants pending (money to institution): AHRQ; Board membership: American Board of Internal Medicine, Salem Hospital; Payment for lectures: More than 100 health care organizations (such as hospitals, health care systems, state medical, and hospital associations); Royalties: Lippincott, Williams \& Wilkins, McGraw-Hill; Payment for development of educational presentations (money to institution): QuantiaMD, In-Patient Consulting-The Hospitalist Company; Stock/stock op-

www.annals.org tions: PatientSafe Solutions, CRISI, EarlySense; Other: John Wiley and Sons, Marc and Lynne Benioff, United States-United Kingdom Fulbright Commission. Ms. McDonald: Grant (money to institution): AHRQ. Dr. Schoelles: Support for travel to meetings and support of work on publication of "Making Health Care Safer II" (money to institution): RAND Corporation (funded by AHRQ). Dr. Dy: Grant (money to institution): AHRQ. Dr. Reston: Grant (money to institution): AHRQ. Dr. Adams: Support for travel to meetings: RAND Corporation. Dr. Bates: Consulting fee and support for travel to meetings: RAND Corporation; Consultancy: PatientSafe Solutions; Royalties: Medicalis; Stocklstock options: Calgary Scientific. Dr. Bickman: Support for travel to meetings and fees for participation in review activities: RAND Corporation. Dr. Carayon: Support for travel to meetings: RAND Corporation; Employment: University of Wisconsin-Madison; Grants/grants pending: AHRQ, Office of the National Coordinator; Royalties: Taylor \& Francis. Dr. Donaldson: Consulting fee and support for travel to meetings: RAND Corporation. Dr. Farley: Grant and support for travel to meetings: AHRQ; Consultancy: RAND Corporation, World Health Organization; Employment: RAND Corporation. Dr. Greenhalgh: Consulting fee and support for travel to meetings: RAND Corporation. Dr. Lake: Consulting fee and support for travel to meetings: RAND Corporation. Dr. Lilford: Grant: National Institute of Health Research Collaborations for Leadership in Applied Health Research and Care for Birmingham and the Black Country; Consulting fee: AHRQ; Support for travel to meetings: AHRQ. Dr. Lohr: Consulting fee: RAND Corporation. Dr. Meyer: Grant: RAND Corporation; Support for travel to meetings (money to institution): RAND Corporation; Expert testimony: Winston Straw. Dr. Miller: Consulting fee: RAND Corporation. Dr. Neuhauser: Consulting fee and support for travel to meetings: RAND Corporation. Dr. Ryan: Grant, consulting fee, support for travel to meetings, fees for participation of review activities, and payment for writing or reviewing the manuscript (money to institution): AHRQ. Dr. Saint: Consulting fee and support for travel to meetings: RAND Corporation (funded by AHRQ); Payment for lectures: Various hospitals, academic medical centers, group-purchasing organizations (for example, Veterans Health Administration and Premier), professional societies (for example, Society of Hospital Medicine), and nonprofit foundations (for example, Institute for Healthcare Improvement and Michigan Health and Hospital Association); Stock/stock options: Doximity. Dr. Shortell: Support for travel to meetings: AHRQ. Dr. Stevens: Consulting fee and support for travel to meetings: RAND Corporation (funded by AHRQ). All other authors have no disclosures. Disclosures can also be viewed at www.acponline.org/authors /icmje/ConflictOfInterestForms.do?msNum=M12-2931.

Requests for Single Reprints: Paul G. Shekelle, MD, PhD, RAND Corporation, 1776 Main Street, Santa Monica, CA 90401; e-mail, shekelle@rand.org.

Current author addresses and author contributions are available at www .annals.org.

\section{References}

1. Shojania KG, Duncan BW, McDonald KM, Wachter RM, Markowitz AJ. Making health care safer: a critical analysis of patient safety practices. Evid Rep Technol Assess (Summ). 2001:i-x, 1-668. [PMID: 11510252]

2. Shekelle PG, Pronovost PJ, Wachter RM, Taylor SL, Dy SM, Foy R, et al. Advancing the science of patient safety. Ann Intern Med. 2011;154:693-6. [PMID: 21576538]

3. Whitlock EP, Lin JS, Chou R, Shekelle P, Robinson KA. Using existing systematic reviews in complex systematic reviews. Ann Intern Med. 2008;148: 776-82. [PMID: 18490690]

4. Shojania KG, Sampson M, Ansari MT, Ji J, Doucette S, Moher D. How quickly do systematic reviews go out of date? A survival analysis. Ann Intern Med. 2007;147:224-33. [PMID: 17638714]

5. Agency for Healthcare Research and Quality. Methods Guide for Effectiveness and Comparative Effectiveness Reviews. AHRQ publication no. 10(11)-

5 March $2013 \mid$ Annals of Internal Medicine $\mid$ Volume 158 • Number 5 (Part 2) $\mid 367$ 
SuPPLEMENT The Top Patient Safety Strategies That Can Be Encouraged for Adoption Now

EHC063-EF. Rockville, MD: Agency for Healthcare Research and Quality; 2011. Accessed at http://effectivehealthcare.ahrq.gov/ehc/products/60/318 /MethodsGuide_Prepublication-Draft_20120523.pdf on 20 July 2012.

6. Cochrane Effective Practice and Organisation of Care Group (EPOC) Reviews. Accessed at http://epoc.cochrane.org/epoc-reviews on 20 July 2012.

7. Harris RP, Helfand M, Woolf SH, Lohr KN, Mulrow CD, Teutsch SM, et al; Methods Work Group, Third US Preventive Services Task Force. Current methods of the US Preventive Services Task Force: a review of the process. Am J Prev Med. 2001;20:21-35. [PMID: 11306229]

8. Higgins JP, Altman DG, Gøtzsche PC, Jüni P, Moher D, Oxman AD, et al; Cochrane Bias Methods Group. The Cochrane Collaboration's tool for assessing risk of bias in randomised trials. BMJ. 2011;343:d5928. [PMID: 22008217]
9. Shekelle PG, Wachter RM, Pronovost PJ, Schoelles K, McDonald KM, Dy SM, et al. Making Health Care Safer II: An Updated Critical Analysis of the Evidence for Patient Safety Practices. (Prepared by the Southern CaliforniaRAND Evidence-based Practice Center under contract HHSA290200710062I.) Rockville, MD: Agency for Healthcare Research and Quality; 2013. [Forthcoming]. 10. Owens DK, Lohr KN, Atkins D, Treadwell JR, Reston JT, Bass EB, et al. AHRQ series paper 5: grading the strength of a body of evidence when comparing medical interventions - agency for healthcare research and quality and the effective health-care program. J Clin Epidemiol. 2010;63:513-23. [PMID: 19595577]

11. Grading of Recommendations Assessment, Development and Evaluation (GRADE) Working Group. Accessed at www.gradeworkinggroup.org on 20 July 2012. 
Current Author Addresses: Dr. Shekelle: Veterans Affairs Greater Los Angeles Healthcare System, 11301 Wilshire Boulevard, Los Angeles, CA 90073.

Dr. Pronovost: Johns Hopkins University School of Medicine, 1909 Thames Street, 2nd Floor, Baltimore, MD 21231.

Dr. Wachter: University of California, San Francisco, 505 Parnassus Avenue, San Francisco, CA 94143.

Ms. McDonald: Stanford University, 117 Encina Commons, Stanford, CA 94305-6019.

Drs. Schoelles and Reston: ECRI Institute, 5200 Butler Pike, Plymouth Meeting, PA 19462-1298.

Dr. Dy: Johns Hopkins University, Room 609, 624 North Broadway, Baltimore, MD 21205.

Dr. Shojania: Sunnybrook Health Sciences Centre, Room H468, 2075 Bayview Avenue, Toronto, Ontario M4N 3M5, Canada.

Dr. Adams: Kaiser Permanente, Division of Research, 2000 Broadway, Oakland, CA 94612.

Dr. Angood: American College of Physician Executives, 400 North Ashley Drive, Suite 400, Tampa, FL 33602.

Dr. Bates: American College of Physician Executives, 400 North Ashley Drive, Suite 400, Tampa, FL 33602.

Dr. Bickman: Center for Evaluation and Program Improvement, Vanderbilt University's Peabody College, Peabody \#151, 230 Appleton Place, Nashville, TN 37203

Dr. Carayon: University of Wisconsin-Madison, 3126 Engineering Centers Building, 1550 Engineering Drive, Madison, WI 53706.

Dr. Donaldson: Department of Surgery \& Cancer, Division of Surgery, Imperial College London, Room 1090a, 10th Floor, QEQM Building, St Mary's Hospital, Praed Street, London W2 1NY, United Kingdom. Dr. Duan: New York State Psychiatric Institute, 1051 Riverside Drive, Unit 48, New York, NY 10032.

Dr. Farley: RAND Corporation, 4570 5th Avenue \#600, Pittsburgh, PA 15213.

Dr. Greenhalgh: Global Health, Policy and Innovation Unit, Centre for Primary Care and Public Health, Blizard Institute, Barts and The London School of Medicine and Dentistry, Yvonne Carter Building, 58 Turner Street, London E1 2AB, United Kingdom.

Dr. Haughom: PeaceHealth, 770 East 11th Avenue, Eugene, OR 97401. Dr. Lake: University of Pennsylvania School of Nursing, Room 302 Fagin Hall, 418 Curie Boulevard, Philadelphia, PA 19104-4217.

Dr. Lilford: University of Birmingham, Room 110, 90 Vincent Drive, Edgbaston, Birmingham B15 2TT, United Kingdom.

Dr. Lohr: RTI International, 3040 Cornwallis Road, PO Box 12194, Research Triangle Park, NC 27709-2194.

Dr. Meyer: Dartmouth-Hitchcock, One Medical Center Drive, Lebanon, NH 03756.

Dr. Miller: Johns Hopkins Children's Center, 200 North Wolfe Street, Room 2094, Baltimore, MD 21287.

Dr. Neuhauser: Case Western Reserve University, 10900 Euclid Avenue, Cleveland, $\mathrm{OH}$ 44106-4945.
Dr. Ryan: RAND Corporation, 1776 Main Street, Santa Monica, CA 90401.

Dr. Saint: Veterans Affairs Ann Arbor Healthcare System, 2215 Fuller Road, Ann Arbor, MI 48105.

Dr. Shortell: University of California, Berkeley, 50 University Hall, MC 7360, Berkeley, CA 94720-7360.

Dr. Stevens: Dartmouth Institute for Health Policy and Clinical Practice, 30 Lafayette Street, Lebanon, NH 03766.

Dr. Walshe: University of Manchester, Booth Street West, Manchester M15 6PB, United Kingdom.

Author Contributions: Conception and design: P.G. Shekelle, P.J. Pronovost, R.M. Wachter, K.M. McDonald, K. Schoelles, S.M. Dy, K. Shojania, J.T. Reston, A.S. Adams, P.B. Angood, D.W. Bates, L. Bickman, P. Carayon, L. Donaldson, N. Duan, D.O. Farley, T. Greenhalgh, J.L. Haughom, E. Lake, R. Lilford, K.N. Lohr, G.S. Meyer, M.R. Miller, D.V. Neuhauser, G. Ryan, S. Saint, S.M. Shortell, D.P. Stevens, K. Walshe.

Analysis and interpretation of the data: P.G. Shekelle, P.J. Pronovost, R.M. Wachter, K.M. McDonald, K. Schoelles, S.M. Dy, K. Shojania, J.T. Reston, A.S. Adams, P.B. Angood, D.W. Bates, L. Bickman, P. Carayon, L. Donaldson, N. Duan, D.O. Farley, T. Greenhalgh, J.L. Haughom, E. Lake, R. Lilford, K.N. Lohr, G.S. Meyer, M.R. Miller, D.V. Neuhauser, G. Ryan, S. Saint, S.M. Shortell, D.P. Stevens, K. Walshe.

Drafting of the article: P.G. Shekelle, P.J. Pronovost, R.M. Wachter. Critical revision of the article for important intellectual content: P.G. Shekelle, P.J. Pronovost, R.M. Wachter, K.M. McDonald, K. Schoelles, S.M. Dy, K. Shojania, J.T. Reston, A.S. Adams, P.B. Angood, D.W. Bates, L. Bickman, P. Carayon, L. Donaldson, N. Duan, D.O. Farley, T. Greenhalgh, J.L. Haughom, E. Lake, R. Lilford, K.N. Lohr, G.S. Meyer, M.R. Miller, D.V. Neuhauser, G. Ryan, S. Saint, S.M. Shortell, D.P. Stevens, K. Walshe.

Final approval of the article: P.G. Shekelle, P.J. Pronovost, R.M. Wachter, K.M. McDonald, K. Schoelles, S.M. Dy, K. Shojania, J.T. Reston, A.S. Adams, P.B. Angood, D.W. Bates, L. Bickman, P. Carayon, L. Donaldson, N. Duan, D.O. Farley, T. Greenhalgh, J.L. Haughom, E. Lake, R. Lilford, K.N. Lohr, G.S. Meyer, M.R. Miller, D.V. Neuhauser, G. Ryan, S. Saint, S.M. Shortell, D.P. Stevens, K. Walshe.

Statistical expertise: K. Shojania, N. Duan, D.V. Neuhauser.

Obtaining of funding: P.G. Shekelle, P.J. Pronovost, R.M. Wachter, K.M. McDonald, K. Schoelles.

Collection and assembly of data: P.G. Shekelle, P.J. Pronovost, R.M. Wachter, K.M. McDonald, K. Schoelles, S.M. Dy, K. Shojania, J.T. Reston, A.S. Adams, P.B. Angood, D.W. Bates, L. Bickman, P. Carayon, L. Donaldson, N. Duan, D.O. Farley, T. Greenhalgh, J.L. Haughom, E. Lake, R. Lilford, K.N. Lohr, G.S. Meyer, M.R. Miller, D.V. Neuhauser, G. Ryan, S. Saint, S.M. Shortell, D.P. Stevens, K. Walshe. 